\title{
Solid conduction effects and design criteria in moving bed heat exchangers
}

\author{
J.A. Almendros-Ibáñez ${ }^{\mathrm{a}, \mathrm{b}, *}$, A. Soria-Verdugo ${ }^{\mathrm{c}}$, U. Ruiz-Rivas $^{\mathrm{c}}$, D. Santana ${ }^{\mathrm{c}}$ \\ ${ }^{a}$ Escuela de Ingenieros Industriales, Dpto. de Mecánica Aplicada e Ingeniería de Proyectos, Castilla La Mancha University, Campus Universitario, 02071 Albacete, Spain \\ ${ }^{\mathrm{b}}$ Renewable Energy Research Institute, Section of Solar and Energy Efficiency, Avda. de la Investigación s/n, 02071 Albacete, Spain

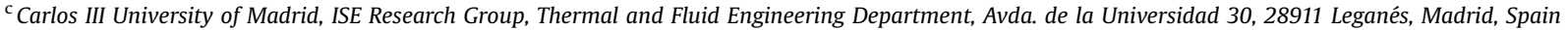

Keywords:

Moving bed heat exchanger

Heat transfer

Biot number

Packed bed

\begin{abstract}
A B S T R A C T
This work presents a theoretical study of the energetic performance of a moving bed heat exchanger (MBHE), which consists of a flow of solid particles moving down that recovers heat from a gas flow percolating the solids in cross flow. In order to define the solid conduction effects, two solutions for the MBHE energy equations have been studied: an analytical solution considering only convection heat transfer (and neglecting solid conduction) and a numerical solution with the solid conductivity retained in the equations. In a second part, the power requirements of a MBHE (to pump the gas and to raise the down flowing particles) are confronted with the heat transferred considering the variation of design parameters, such as gas and solids' velocities, solids particle diameter or MBHE dimensions.

The numerical results show that solid conductivity reduces the global efficiency of the heat exchanger. Therefore, a selection criterion for the solids can be established, in which their thermal conductivity should be minimized to avoid conduction through the solid phase, but to a limit in order to ensure that temperature differences inside an individual solid particle remain small. Regarding the other energy interactions involved in the system, these are at least one order of magnitude lower than the heat exchanged. Nevertheless, for a proper analysis of the system the efficiency of the devices used to pump the gas and to raise the particles and the relative costs of the different energy forms present in the system should be taken into account.
\end{abstract}

\section{Introduction}

Moving bed heat exchangers (MBHEs hereafter, and often called packed bed heat exchangers) are widely used in industry, for applications involving heat recovery, solid drying, filtering or thermochemical conversion processes. Compared with other systems, they provide a large heat transfer area in a reduced volume and, concerning filtering, they avoid common operational problems that are typical of fixed bed or ceramic filters, such as the pressure drop increase during operation.

Several studies can be found in the literature concerning flow patterns and particles velocity in moving beds, as for example the works by Hsiau et al. [1-3] as well as on the heat transfer between gas and particles in fixed or moving beds [4-9]. Moving beds are often found in heat recovery systems, like the usual counter flow regenerator that transfers heat between two fluid flows. Also, they

\footnotetext{
* Corresponding author. Escuela de Ingenieros Industriales, Dpto. de Mecánica Aplicada e Ingeniería de Proyectos, Castilla La Mancha University, Campus Universitario, 02071 Albacete, Spain. Tel.: +34967599200.

E-mail address: jose.almendros@uclm.es (J.A. Almendros-Ibáñez).
}

can be used to recover heat from a flow of solids to another flow of solids [10] or to dry a flow of solids [11]. On the other hand, different equipments have been proposed for hot gas particulate removal, such as electrostatic precipitators, ceramic filters, scrub bers, bag filters and granular filters $[1,4,12,13]$. Smid et al. [14] made a complete review of the patent literature about moving bed filters and their equipment in different countries around the world. MBHE are increasing in interest as a key component in integrated gasifi cation combined cycles, as well as in pressurized fluidized bed combustors, due to two main advantages: their capacity to properly filter the gas stream at high temperatures and their suitability to be used also as heat exchangers. More recently, MBHE has also been employed in novel thermochemical conversion processes for the production of uranium tetrafluoride [15] or for catalytic naphtha reforming [16].

The bed material used in the MBHE depends on the application. For high temperature heat exchange and filtration, alumina and silica sand (with a size ranging between 0.5 and $2.0 \mathrm{~mm}$ ) are typically used in industrial applications $[13,17]$. Spheres of steel are also widely used [6,9]. Recently, Macias Machin et al. [18] presented "lapilly", a new material for gas filtration applications. In applications different to heat recovery and gas filtration specific 
Table 1

Experimental data of the work of Henriquez and Macías-Machín [9]. Variables that are subject to variations throughout the paper are in bold format and capital letters.

\begin{tabular}{|c|c|}
\hline GAS INLET TEMPERATURE, $T_{\text {in }}$ & $100 \circ \mathrm{C}$ \\
\hline Solid inlet temperature $\theta_{\text {in }}$ & $25^{\circ} \mathrm{C}$ \\
\hline GAS VELOCITY, $u_{\mathrm{g}}$ & $1.5 \mathrm{~m} / \mathrm{s}$ \\
\hline SOLID VELOCITY, $u_{\mathrm{s}}$ & $5 \mathrm{~cm} / \mathrm{min}$ \\
\hline Specific heat of the gas, $c_{\mathrm{p}, \mathrm{g}}$ & $1005 \mathrm{~J} /(\mathrm{kgK})$ \\
\hline Specific heat of the solids, $c_{\mathrm{s}}$ & $544 \mathrm{~J} /(\mathrm{kgK})$ \\
\hline Gas density, $\rho_{g}$ & $1 \mathrm{~kg} / \mathrm{m}^{3}$ \\
\hline Solid density, $\rho_{S}$ & $7800 \mathrm{~kg} / \mathrm{m}^{3}$ \\
\hline Gas conductivity, $k_{\mathrm{g}}$ & $0.03 \mathrm{~W} /(\mathrm{mK})$ \\
\hline SOLID CONDUCTIVITY, $k_{\mathrm{s}}$ & $15 \mathrm{~W} /(\mathbf{m K})$ \\
\hline Gas dynamic viscosity, $\mu_{\mathrm{g}}$ & $2.12 \times 10{ }^{5} \mathrm{Pas}$ \\
\hline PARTICLE DIAMETER, $d_{\mathrm{p}}$ & $10^{-3} \mathrm{~m}$ \\
\hline LENGTH IN THE DIRECTION OF THE GAS FLOW, $L$ & $0.15 \mathrm{~m}$ \\
\hline HEIGHT IN THE DIRECTION OF THE SOLIDS FLOW, $\mathrm{H}$ & $0.5 \mathrm{~m}$ \\
\hline Bed porosity, $\varepsilon$ & 0.4 \\
\hline Ratio of gas specific heats, $\gamma$ & 1.4 \\
\hline Wall porosity, $\varepsilon_{\mathrm{W}}$ & 0.5 \\
\hline Gas pressure at the inlet section, $P_{\text {in }}$ & $10^{5} \mathrm{~Pa}$ \\
\hline
\end{tabular}

materials can be used. For example, Niksiar and Rahimi [15] reduced granulated uranium trioxide in a moving bed during the process to obtain uranium tetrafluoride.

This article focuses on the design of a MBHE based on energy criteria (increasing heat transfer without dramatically increasing the power consumption needed to move the two flows) with emphasis on solid conduction effects. On a previous article [19] we presented an exergy analysis of the MBHE, in which an optimized length (in the fluid flow direction) and solid particle diameter were obtained. In the following, the relative importance of conduction in the solid phase is analyzed and different approaches are presented to define the adequate parameters (particle diameter and the velocities of both flows) for a given application.

In the results showed along this article, the fluid is air and there is no mass transfer between fluid and solids (i.e. no solids drying, filtering or phase change). The nominal values of the data are obtained from the experimental set up of Henriquez and Macías Machín [9], which are summarized in Table 1. The properties of the solids showed in this table correspond with the properties of the spheres of steel used by Henriquez and Macías Machín [9].

Fig. 1(a) shows a general scheme of the MBHE geometry. The solids move down in the positive $y$ direction and the gas percolates through the solid particles in cross flow (moving from left to right), in the positive $x$ direction. In the following we will assume the 2D geometry showed in Fig. 1(b) for the heat transfer analysis.

\section{Governing equations}

The general two phase equations governing heat transfer for the MBHE shown in Fig. 1(a) are given by the equation system (1) and (2). Heat losses to the surroundings, radiation heat transfer and the loss of solids' potential energy are neglected in this analysis.

$$
\begin{aligned}
& \varepsilon \rho_{\mathrm{g}} c_{\mathrm{p}, \mathrm{g}}\left(\frac{\partial T}{\partial t}+\boldsymbol{u}_{\mathrm{g}} \cdot \nabla T\right) \quad \nabla \cdot\left(k_{\mathrm{g}} \nabla T\right)+h_{\mathrm{s}} a_{\mathrm{s}}\left(\begin{array}{ll}
\theta & T)
\end{array}\right.
\end{aligned}
$$

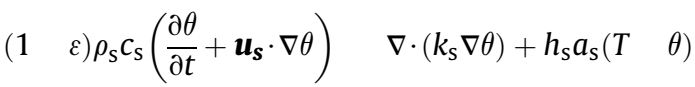

Tand $\theta$ are the gas and solid temperature, respectively, and $a_{\mathrm{s}}$ is the superficial particle area per unit of volume. When the fluid used in the MBHE is a gas the diffusion term of Eq. (1) is usually orders of magni tude lower than the convective term and can be neglected in the calculations. In contrast the solid conductivity, especially for metallic materials, cannot be neglected and it is retained in the calculations.

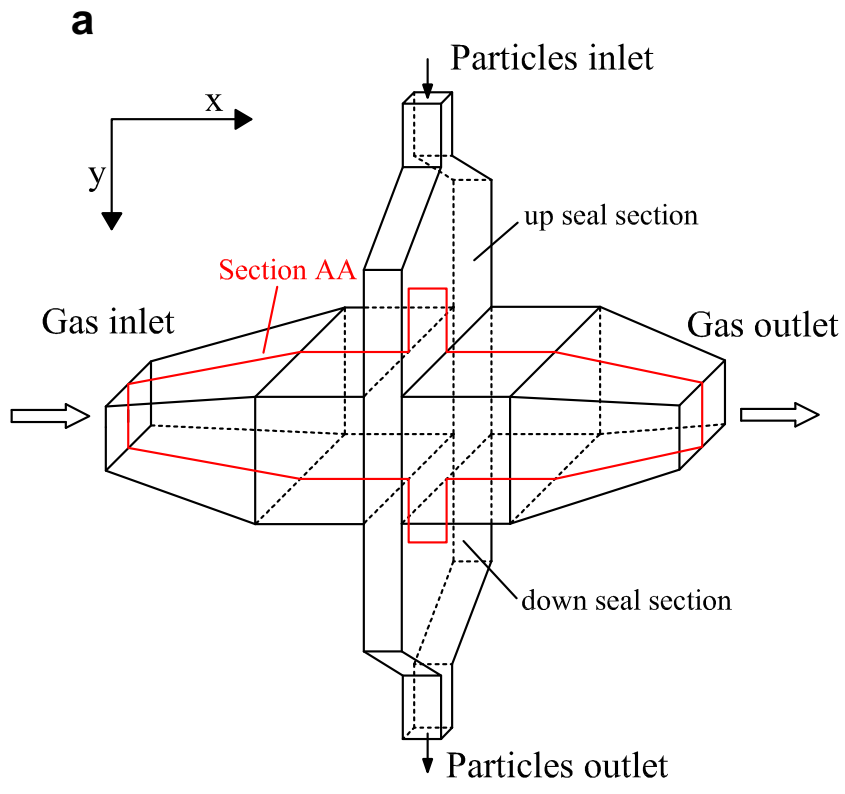

General view

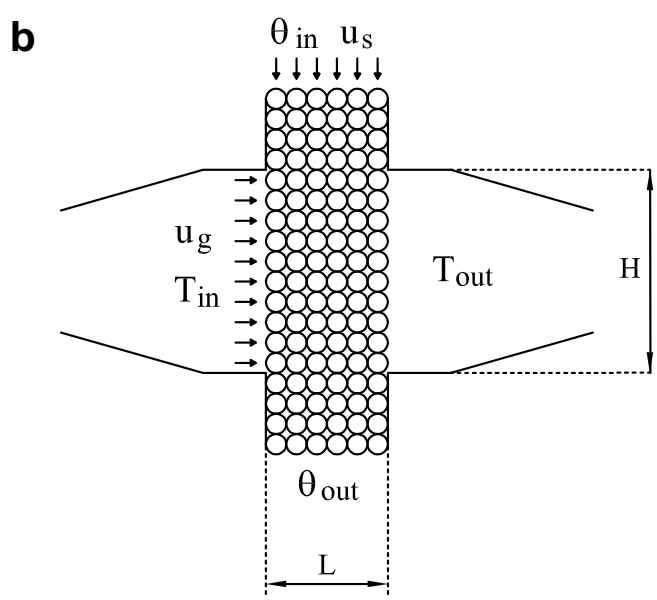

View of section AA

Fig. 1. Schematic of a moving bed heat exchanger (MBHE).

A common simplification in MBHE modeling is the assumption of plug flow in both phases (gas and solids), which is equivalent to assuming a constant voidage in the bed and uniform velocity profiles for both phases. This is not true close to the walls of the bed, so a much more complex modeling is needed for narrow beds. A number of works have studied the particles and gas flow close to the walls, both numerically and experimentally, in order to state its characteristics and relevance. The MiDi research group [20] analyzed the behavior of dense assemblies of dry grains submitted to continuous shear deformation. For vertical chute flows, where gravity drives the material down between the walls, both particle velocity profile and bed porosity profile are charac terized by a plug region in the central part of the channel and shear zones near the walls, where particle velocity and porosity vary. The thickness of such zones is of the order of 5-6 particle diameters for nearly spherical particles. This is also in agreement with the work of Nedderman and Laohakul [21]. They also showed that the particle velocity at the walls is $25 \%$ below the velocity in the plug flow region for fully rough walls. Zou and Yu [22] showed, for both loose 
and dense packing, that at a distance of 2.5 particle diameters from the wall, the mean wall porosity was approximately $10 \%$ above the bed core porosity. In contrast, Takahashi and Yanai [23] obtained experimentally that only 2-3 column diameters are needed to reach a stable plug flow region. Moreover, they observed that, although the bed porosity is slightly influenced by the velocity of the descending particles, this influence vanishes when the solid flow rate increases. Finally, their results showed that particle velocity at wall is 0.7 to 0.8 the mean particle velocity at bed core. Van Antwerpen et al. [24] have recently reviewed the correlations to model the bed porosity and the effective thermal conductivity in packed beds, showing that the local porosity behavior near the wall is similar to a damped harmonic oscillator, whereas the porosity, averaged by a particle diameter, can be approximated by an expo nential function. Previously, Giese et al. [25] proved, for different particle sizes, that the local gas velocity near the wall has the same damped harmonic behavior as the local bed porosity. But once again the local gas velocity oscillation damped when the Reynolds particle number increases.

On the other hand, several works have dealt with the gas distri bution in a moving bed and the plug flow assumption for the gas phase. Vortmeyer and Winter [26] reviewed experimental findings on the homogeneous behavior of packed beds and concluded that the limit of homogeneity lies much lower than predicted by a purely mathematical reasoning. They suggested that the limiting bed/ particle diameter ratio must be at least 4 to consider gas plug flow in the packed bed. They concluded that, for their experimental condi tions (ratios between the reactor diameter and the particle size between 2 and 3 ), it was not necessary to extend the modeling by adding a variation in the radial or axial dispersion coefficient in the gas phase. On the other hand, Teplitskii et al. [27] showed that the thickness ratio between the filtration boundary layer and the viscous boundary layer is 1.78 , the same ratio was found for the thickness of the filtration thermal boundary layer and the thermal sublayer. Therefore, the ratio of the filtration boundary layer respect to particle diameter being equal to $0.33 \mathrm{Re}_{\mathrm{p}}^{-0.31}$ for $\operatorname{Re}_{\mathrm{p}}>120$ the thickness of both boundary layers are lower than a particle diameter. Also, and for Prandtl number of order unity, the thickness of the filtration thermal boundary layer and the thermal sublayer are of the same order of the particle diameter. Another source of gas maldistribution can be attributed to the cavity and pinning phenomena [28]. For a cross flow, the particles close to the upstream face may leave the face and a cavity forms between the upstream face and the granular bed, while in the downstream face, the frictional force can be enough to stop the particles, forming a dead zone (pinning). Nevertheless, these two phenomena are only relevant for extremely high gas velocities.

In view of these findings [20-28], the assumption of plug flow for both solid and gas phase will be considered acceptable if the dimensions of the bed are larger than 10 times the particle diam eter. Therefore, assuming a 2D geometry (see Fig. 1) and steady state conditions, the governing Eqs. (1) and (2) can be written in compact and non dimensional form as

$$
\frac{\partial \widehat{\theta}}{\partial \eta} \quad K_{\xi} \frac{\partial^{2} \widehat{\theta}}{\partial \xi^{2}} \quad K_{\eta} \frac{\partial^{2} \widehat{\theta}}{\partial \eta^{2}} \quad \widehat{T} \quad \widehat{\theta} \quad \frac{\partial \widehat{T}}{\partial \xi},
$$

where

$$
\widehat{\theta} \frac{\theta \quad \theta_{\text {in }}}{T_{\text {in }} \theta_{\text {in }}}, \widehat{T} \quad \frac{T}{T_{\text {in }} \theta_{\text {in }}}
$$

are the non dimensional temperatures, varying between 0 and 1 ,

$$
\xi \quad \frac{x h_{\mathrm{s}} a_{\mathrm{s}}}{\varepsilon \rho_{\mathrm{g}} \mathrm{u}_{\mathrm{g}} c_{\mathrm{p}, \mathrm{g}}}, \eta \quad \frac{y h_{\mathrm{s}} a_{\mathrm{s}}}{(1 \quad \varepsilon) \rho_{\mathrm{s}} u_{\mathrm{s}} c_{\mathrm{s}}}
$$

are the non dimensional horizontal ( $x$ direction) and vertical ( $y$ direction) coordinates, respectively, and

$$
K_{\xi} \quad \frac{h_{\mathrm{s}} a_{\mathrm{s}} k_{\mathrm{s}, x}}{\left(\varepsilon \rho_{\mathrm{g}} u_{\mathrm{g}} c_{\mathrm{p}, \mathrm{g}}\right)^{2}}, K_{\eta} \quad \frac{h_{\mathrm{s}} a_{\mathrm{s}} k_{\mathrm{s}, y}}{\left(\left(\begin{array}{ll}
1 & \varepsilon
\end{array}\right) \rho_{\mathrm{s}} u_{\mathrm{s}} c_{\mathrm{s}}\right)^{2}}
$$

are the non dimensional conductivities in the direction of the gas flow and in the direction of the solid flow, respectively. The value of the thermal conductivity in the direction of the gas flow was obtained using the correlation proposed by Krupiczka [29], and the thermal conductivity in the direction of the particle flow using the equation presented by Yagi et al. [6], as suggested by Marb and Vortmeyer [30]. The convection heat transfer coefficient was obtained with a correlation proposed by Achenbach [8].

In order to solve the non dimensional equation system (3) a set of boundary conditions is needed. The one shown in Table 2 are considered to properly state the underlying physics, as shown by Marb and Vortmeyer [30]. The differential equation system (3) can be solved numerically using a finite difference technique. The elliptic character of the equations is transformed into parabolic adding a temporal derivative into the solid equation. The first derivatives are discretized using an up wind scheme and the second derivatives using central differences. A more detailed description of the numerical scheme can be seen in [19].

The equation system (3) has also an analytical solution when the conduction terms are negligible, which is usually accepted for high Reynolds numbers [31]. Then, the equation system (3) becomes:

$$
\begin{array}{llll}
\frac{\partial \hat{\theta}}{\partial \eta} & \widehat{T} & \widehat{\theta} & \frac{\partial \widehat{T}}{\partial \xi}
\end{array}
$$

The two boundary conditions needed to solve the equation system (7) are

$$
\widehat{T}_{\xi} \quad 0 \quad 1 \text { and } \widehat{\theta}_{\eta} 0 \quad 0 \text {. }
$$

With such conditions, according to Saastamoinen [32] and previous authors, the analytical solution for the non dimensional gas and solid temperatures are

$$
\begin{aligned}
& \widehat{T} \quad e^{-\xi-\eta} \sum_{j}^{\infty} \frac{\eta^{j}}{j !} \sum_{k}^{j} \frac{\xi^{k}}{k !} \\
& \widehat{\theta} \quad 1 \quad e^{-\eta-\xi} \sum_{j}^{\infty} \frac{\xi^{j}}{j !} \sum_{k}^{j} \frac{\eta^{k}}{k !}
\end{aligned}
$$

\section{Analysis}

\subsection{Heat transfer and conduction effects}

First we will discuss the heat transfer issues and the relative importance of conduction effects. As shown in Soria Verdugo et al.

\section{Table 2}

Boundary conditions for solving equation system (3).

\begin{tabular}{lllll}
\hline Particles & $\xi$ & 0 & $\frac{\partial^{2} \widehat{\theta}}{\partial \xi^{2}}$ & 0 \\
& $\xi$ & $\xi_{L}$ & $\frac{\partial^{2} \widehat{\theta}}{\partial \xi^{2}}$ & 0 \\
& $\eta$ & 0 & $\widehat{\theta}$ & $K_{\eta} \frac{\partial \widehat{\theta}}{\partial \eta}$ \\
Gas & $\eta$ & $\eta_{H}$ & $\frac{\partial \widehat{\theta}}{\partial \eta}$ & 0 \\
\hline
\end{tabular}


Table 3

Non-dimensional parameters obtained with the nominal data of Henriquez and Macias-Machin [9].

\begin{tabular}{llllll}
\hline$T_{\text {in }}$ & $\theta_{\text {in }}$ & $\xi_{x=L}$ & $\eta_{y=H}$ & $K_{\xi}$ & $K_{\eta}$ \\
\hline 1 & 0 & 363 & 344 & 51.12 & 4.15 \\
\hline
\end{tabular}

[19] in a study that neglected solid conduction effects, the heat transfer process is optimized for both flows when

$\xi_{x} L \quad \eta_{y} H$

If one of the non dimensional parameters of this equation is larger than the other, a certain part of the flow coming perpen dicular to the larger length will exit the MBHE barely undisturbed (with a temperature near to its inlet temperature). Therefore, Eq. (11) should be fulfilled for a proper heat exchange (or it may not when other issues are of paramount importance, such as a proper filtering). This will be denoted as a "square" MBHE throughout the article, being square only in this non dimensional sense. Going back to Eq. (5) and using typical velocities and properties for solids and air, it can be stated that this square condition usually means that the $H$ dimension is three times larger than the $L$ dimension, with possible variations ranging from almost equal values for both dimensions, to $H 10$ times larger than $L$. Rearranging Eq. (11), also states that the product of the mass flow and the specific heat should be equal for both flows (fluid and solids), a typical result in heat exchangers.

$\dot{m}_{\mathrm{s}} c_{\mathrm{s}} \quad \rho_{\mathrm{s}}(1 \quad \varepsilon) L B u_{\mathrm{s}} c_{\mathrm{s}} \quad \rho_{\mathrm{g}} \varepsilon H B u_{\mathrm{g}} c_{\mathrm{p}, \mathrm{g}} \quad \dot{m}_{\mathrm{g}} c_{\mathrm{p}, \mathrm{g}}$

Moreover, Eq. (12) defines the ratio between MBHE length and height as a function of solid and fluid velocities, and of general properties, giving variations as stated above. Note that the two non dimensional lengths of Eq. (11) are not supposed to be limited by unity. With the previously mentioned MBHE of Henriquez and Macías Machín [9], which represents a rather small MBHE, both non dimensional lengths are around 350. Table 3 shows the non dimensional parameters obtained with the nominal data of Henriquez and Macias Machín [9] summarized in Table 1.

Fig. 2(a) shows the non dimensional gas temperature profiles, when solid conductivity is neglected, for a "square" heat exchanger of the same size of our nominal MBHE. In this situation, an analytical solution (Eq. (9)) can be used. Heat is only transferred by convection from the hot gas to the cold solids in a narrow region of the bed.

Now we will consider solid conduction effects. Fig. 2(b) shows the non dimensional gas temperature profiles for the nominal data showed in Table 1 diminishing the solid conductivity one order of magnitude (from $k_{\mathrm{s}} 15 \mathrm{~W} /(\mathrm{mK})$ to $k_{\mathrm{s}} 1.5 \mathrm{~W} /(\mathrm{mK})$ ), which results in non dimensional conductivities of $K_{\xi} \quad 5.41$ and $K_{\eta} \quad 0.46$. Fig. 2(c) shows the same curves for the nominal data $\left(k_{\mathrm{s}} 15 \mathrm{~W} /(\mathrm{mK})\right)$. In contrast to Fig. 2(a), when conduction is taken into account, part of the heat is transferred by conduction through the solid phase. As a result, the width of the region where $T$ and $\theta$ change increases. Higher conductivities $K_{\xi}$ and $K_{\eta}$ imply larger regions of temperature variation. Also, the different boundary conditions applied to both flows when conduction is considered change the symmetry of the problem (although in a feeble way), as can be seen in the inlet and outlet of particles in Fig. 2(c). Nevertheless, symmetry is still important and Eq. (11) can be used as an adequate design criteria.

The variations between Fig. 2(a)-(c) can be largely attributed to the conduction term in the gas flow direction, $K_{\xi}$, which is roughly an order of magnitude larger than the conduction term in the perpendicular direction $K_{\eta}$. This is a consequence of $H$ being larger
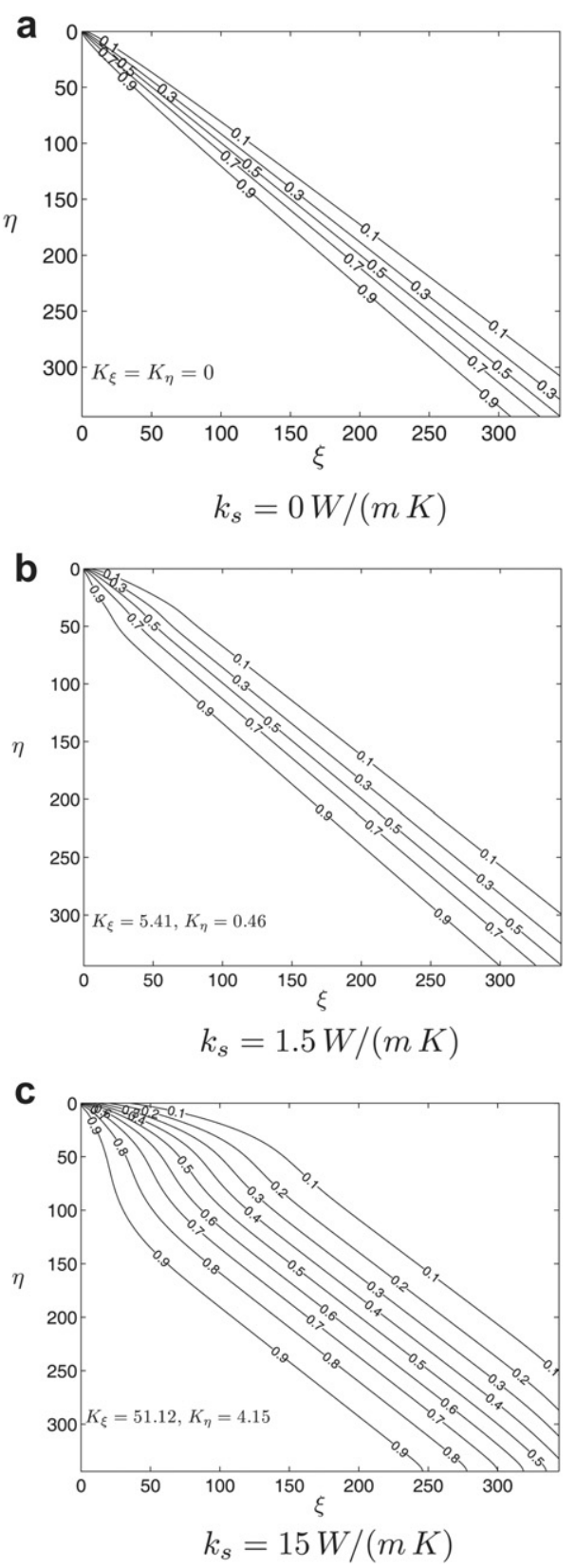

Fig. 2. Non-dimensional gas temperature profiles for the nominal MBHE. (a) Analytical solution for $k_{\mathrm{s}} \quad 0$, (b) numerical solution for $k_{\mathrm{s}} 1.5 \mathrm{~W} /(\mathrm{mK})$ and (c) numerical solution for the nominal case $\left(k_{\mathrm{s}} 15 \mathrm{~W} /(\mathrm{mK})\right)$.

than $L$, as stated above, because when the restriction of Eq. (12) is used over the definitions of Eq. (6), it follows that

$\frac{K_{\xi}}{K_{\eta}} \quad \frac{k_{s, x}}{k_{s, y}}\left(\frac{H}{L}\right)^{2} \sim\left(\frac{H}{L}\right)^{2} \gg 1$.

The main feature of solid conduction is that the net heat transfer between solids and fluid diminishes, as the initially hot flow (an air flow in this case) exits the MBHE with a higher mean outlet temperature in Fig. 2(c) than in Fig. 2(a), due to the larger heat exchange region. Fig. 2(b) shows an intermediate case. This is a rather surprising result, as a higher capability to conduct heat results in a global heat transfer decrease. Therefore, avoiding conduction effects in the solids will maximize the heat transferred by the MBHE. 


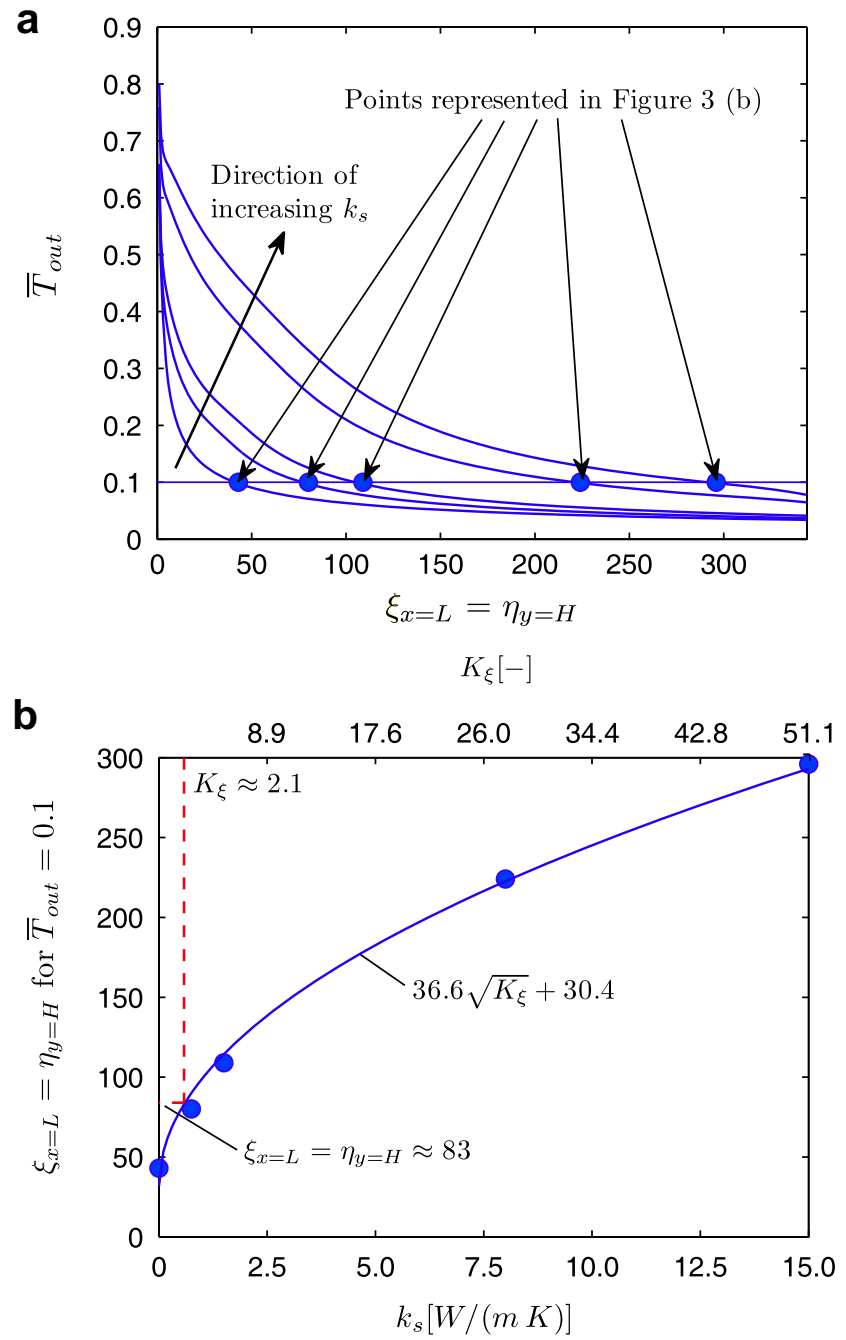

Fig. 3. (a) Non-dimensional mean outlet gas temperature for different solid conductivities ( $\left.k_{\mathrm{s}} \quad 0 \quad 0.75 \quad 1.5 \quad 8 \quad 15 \mathrm{~W} /(\mathrm{m} / \mathrm{K})\right)$ and $(\mathrm{b}) \xi_{x=L}$ for and optimum heat exchanger $\left(\begin{array}{ll}\xi_{x=L} & \eta_{y=H}\end{array}\right)$ with an efficiency of $90 \%\left(\bar{T}_{\text {out }} \quad 0.1\right)$ for different solid conductivities.

Fig. 3(a) shows the non dimensional mean outlet temperature of the gas flow $\bar{T}_{\text {out }}$ as a function of the MBHE non dimensional length, for a "square" MBHE that follows Eq. (11), and for five different conductivities, ranging between $k_{\mathrm{S}} 0$ (neglecting conduction) and $k_{\mathrm{s}} 15 \mathrm{~W} /(\mathrm{mK})$ (our nominal case). This temper ature is directly related with the efficiency of the MBHE, defined as $\varepsilon_{\text {MBHE }} \quad \dot{Q} / \dot{Q}_{\max }$, by Eq. (14)

$\bar{T}_{\text {out }} \quad 1 \quad \varepsilon_{\text {MBHE}}$.

Thus, a value of $\bar{T}_{\text {out }}$ close to zero implies a higher efficiency of the MBHE. The differences between zero, small, medium and large conduction terms are evident. The smaller solid conductivity cases show a fairly similar response than that of the non conduction case for lengths larger than 100. But for the larger conduction case, the length should be larger than 350 to consider that the conduction effect has a feeble impact in the MBHE behavior.

Therefore, for small values of the non dimensional lengths $\xi_{x} L \quad \eta_{y}{ }_{H}$, conduction effects may prove important in diminishing the heat transferred in the MBHE. The effect can be minimized by reducing the conduction term (Eq. (6)). As a consequence, if the influence of conduction heat transfer in the MBHE is more relevant, the size of the heat exchanger should increase to maintain its efficiency. Fig. 3(b) shows the increase of the non dimensional length of the MBHE for different solid conductivities with a heat exchanger efficiency of $\varepsilon_{\text {MBHE }} \quad 90 \%\left(\bar{T}_{\text {out }} \quad 0.1\right)$.

Once more, it might seem rather strange to diminish the solid conductivity in a heat exchanger. Of course, a certain conductivity is needed in order to ascertain that the solids' surface temperature and its inner temperature are similar, so that the convection heat transfer is not affected. Else, the heat transfer would be controlled by solid conduction inside the particles. In order to avoid this effect, general theory [33] states that the Biot number should be

Bi $\quad \frac{h_{\mathrm{s}} d_{\mathrm{p}}}{k_{\mathrm{s}}} \leq 0.1$

Nevertheless, Eq. (15) can be fulfilled for rather small values of the solid conductivity, considering small particles and small convection coefficients based on the air particle interaction.

Fig. 4(a) shows a contour plot of the conduction term in the gas flow direction as a function of gas velocity and particle diameter for the steel spheres considered in the nominal case. Note that the convection coefficient depends on both gas velocity and particle size, increasing with the gas velocity and decreasing when the particle size is increased. In view of this figure, in order to diminish the non dimensional size of the MBHE (or to increase its efficiency with the same non dimensional size), we could increase the particle size and/or the gas velocity, obtaining lower values of $K \xi$.

Fig. 4(b) shows a contour plot of the Biot number. In the range of particle sizes and gas velocity tested, the Biot number always fulfils Eq. (15). Thus, another parameter that can be modified to increase the efficiency or decrease the size (if particle diameter and fluid velocity are fixed) is the conductivity of the solid media used in the MBHE $\left(k_{s}\right)$. This material property affects both Biot number and non dimensional conductivity $K_{\xi}$. Fig. 4(c) shows the data of $K_{\xi}$, as a function of the gas velocity and the particle diameter, and for the minimum value of the solid conductivity that fulfils Eq. (15). Fig. 4(d) shows the contour plot of that minimum solid conductivity.

Therefore, increasing particle size and gas velocity and decreasing solid conductivity, the non dimensional conductivity $K_{\xi}$ is reduced. This fact results in an improvement of the heat exchanger efficiency or in a reduction of its non dimensional size. But note that a reduction of the non dimensional size does not directly lead to a reduction of the actual size. This might be the case when varying $d_{\mathrm{p}}$ and $u_{\mathrm{g}}$, as those two parameters are also involved in the definitions of the non dimensional lengths (Eq. (5)). There fore, the effect on the actual size is not straightforward and should be studied in each case. In contrast, the solid conductivity $k_{s}$ only affects the non dimensional conductivities but not any other parameter in the non dimensional dimensions. Thus, a reduction of $k_{\mathrm{s}}$ implies directly a reduction of the actual MBHE size maintaining the rest of the parameters constant.

For example, point A in Fig. 4(a) and (b) represents the nominal MBHE of Henriquez and Macías Machín [9]. If we increase the size of the particles from $d_{\mathrm{p}} \quad 1 \mathrm{~mm}$ to $d_{\mathrm{p}} \quad 5 \mathrm{~mm}$ with the same gas velocity, the non dimensional solid conductivity $K_{\xi}$ is reduced from $K_{\xi} \approx 51.12$ to $K_{\xi} \approx 4$ (point B in Fig. $4(\mathrm{a})$ ). Consequently, the Biot number increases from $\mathrm{Bi} \quad 0.027$ to $\mathrm{Bi} \approx 0.053$ (point $\mathrm{B}$ in Fig. 4 (b)), always fulfilling Eq. (15). As commented previously, the efficiency of the MBHE can be further increased reducing the solid conductivity. According to the data summarized in Table 1, Henri quez and Macías Machín [9] used particles with a solid conduc tivity of $k_{\mathrm{s}} 15 \mathrm{~W} /(\mathrm{mK})$. Reducing this solid conductivity to $k_{\mathrm{s}} \approx 7.0 \mathrm{~W} /(\mathrm{mK})$ (see point $\mathrm{C}$ in Fig. $4(\mathrm{~d})$ ) the non dimensional conductivity $K_{\xi}$ is reduced to a value of $K_{\xi} \approx 2.1$ (see point C in Fig. 4 (c)) in the limit of Bi 0.1. Finally, introducing this data in Fig. 3(b) 

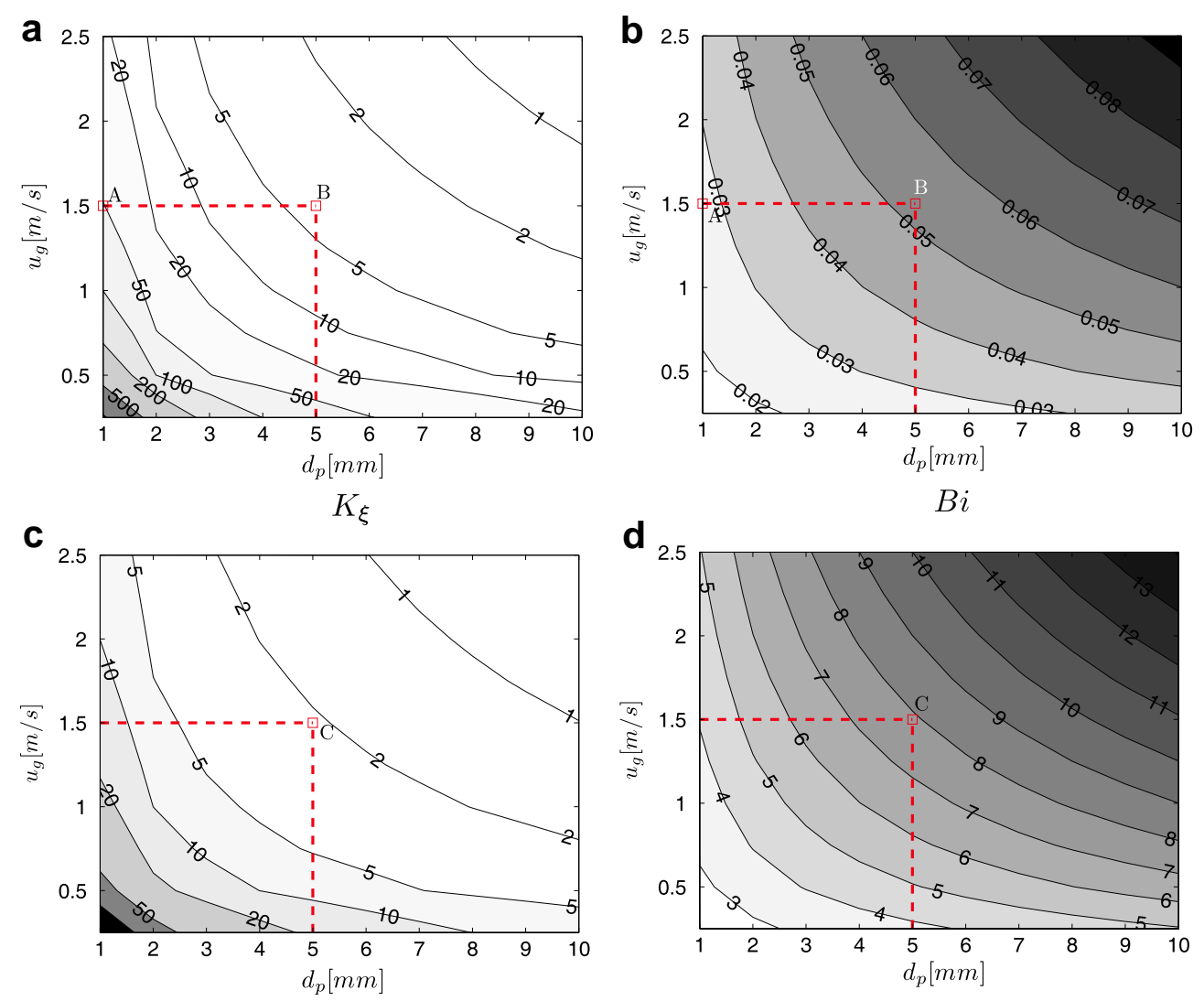

$K_{\xi}$ for $B i=0.1$

$$
k_{s} \text { for } B i=0.1 \text {. Units in } \mathrm{W} /(\mathrm{m} \mathrm{K})
$$

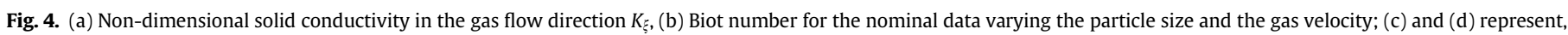
respectively, the non-dimensional conductivity $K_{\xi}$ and the solid conductivity $k_{\mathrm{s}}$ in the limit case of $\mathrm{Bi} \quad 0.1$.

we can check how the non dimensional size needed to exchange $90 \%$ of the maximum heat is reduced to a value $\xi_{x} \quad \approx 83$ (with the original data of Henriquez and Macías Machín [9] a length of $\xi_{x L} \approx 300$ is needed).

\subsection{Heat transfer and power requirements}

After this purely thermal analysis, one should take into account the other thermodynamic interactions, such as the power require ments to pump the fluid through the bed and to raise the solids.

For any MBHE, the heat transferred between gas and particles and the power consumed to pump the gas can be calculated per unit of gas mass flow with Eqs. (16) and (17), while the power required to raise the particles per unit of solid mass flow is expressed according Eq. (18).

$\frac{\dot{Q}}{\dot{m}_{\mathrm{g}}} \quad c_{\mathrm{p}, \mathrm{g}}\left(\bar{T}_{\text {in }} \quad \bar{T}_{\text {out }}\right)$

$\frac{\dot{W}_{\mathrm{g}}}{\dot{m}_{\mathrm{g}}} \quad \frac{\Delta P}{\rho_{\mathrm{g}}}$,

$\frac{\dot{W}_{\mathrm{s}}}{\dot{m}_{\mathrm{s}}} \quad g H$

The gas pressure drop $\Delta P$ can be obtained from Ergun equation [34]:
$\Delta P$

$$
\rho_{\mathrm{g}}\left(\frac{1 \varepsilon}{\varepsilon^{3}}\right) \frac{L}{d_{\mathrm{p}}} u_{\mathrm{g}}^{2}\left(\frac{150(1 \quad \varepsilon) \mu_{\mathrm{g}}}{d_{\mathrm{p}} \rho_{\mathrm{g}} u_{\mathrm{g}}}+1.75\right) .
$$

Although Ergun equation was obtained for fixed (instead of moving) beds, the solids velocity in a MBHE is orders of magnitude lower than the gas velocity. Therefore, the particle movement can be neglected for pressure drop calculation in the majority of MBHE applications.

Eq. (18) can be modified for a "square" MBHE taking into account Eq. (12) as follows

$\frac{\dot{W}_{\mathrm{s}}}{\dot{m}_{\mathrm{g}}} \quad g \frac{\rho_{\mathrm{s}}}{\rho_{\mathrm{g}}}\left(\frac{1 \varepsilon}{\varepsilon}\right) L \frac{u_{\mathrm{s}}}{u_{\mathrm{g}}}$

Eq. (20) has been expressed per unit of gas mass flow (instead of solids flow) to be in concordance with Eqs. (16) and (17).

The results of the power requirements (Eqs. (17) and (20)) and heat transfer (Eq. (16)) per unit of gas mass flow and for "square" heat exchanger are presented in Fig. 5 as a function of the relevant parameters. The heat transfer is a function of the maximum temperature difference and of the non dimensional length (that defines $\bar{T}_{\text {out }}$, as shown in Fig. 3(a)). The heat transferred is shown both assuming or neglecting conduction effects. The power consumed to pump the gas is a function of particle diameter and gas velocity, and the power consumed to raise the particles is a function of the gas and solids velocities. The rest of the parame ters $\left(\begin{array}{lll}L & 0.15 \mathrm{~m}, \varepsilon & 0.4\end{array}\right)$ and properties (both densities, $\rho_{s}$ and $\rho_{g}$, gas specific heat $c_{\mathrm{p}, \mathrm{g}}$ and the dynamic viscosity $\mu_{\mathrm{g}}$ ) are taken from the nominal case (see Table 1 ). 

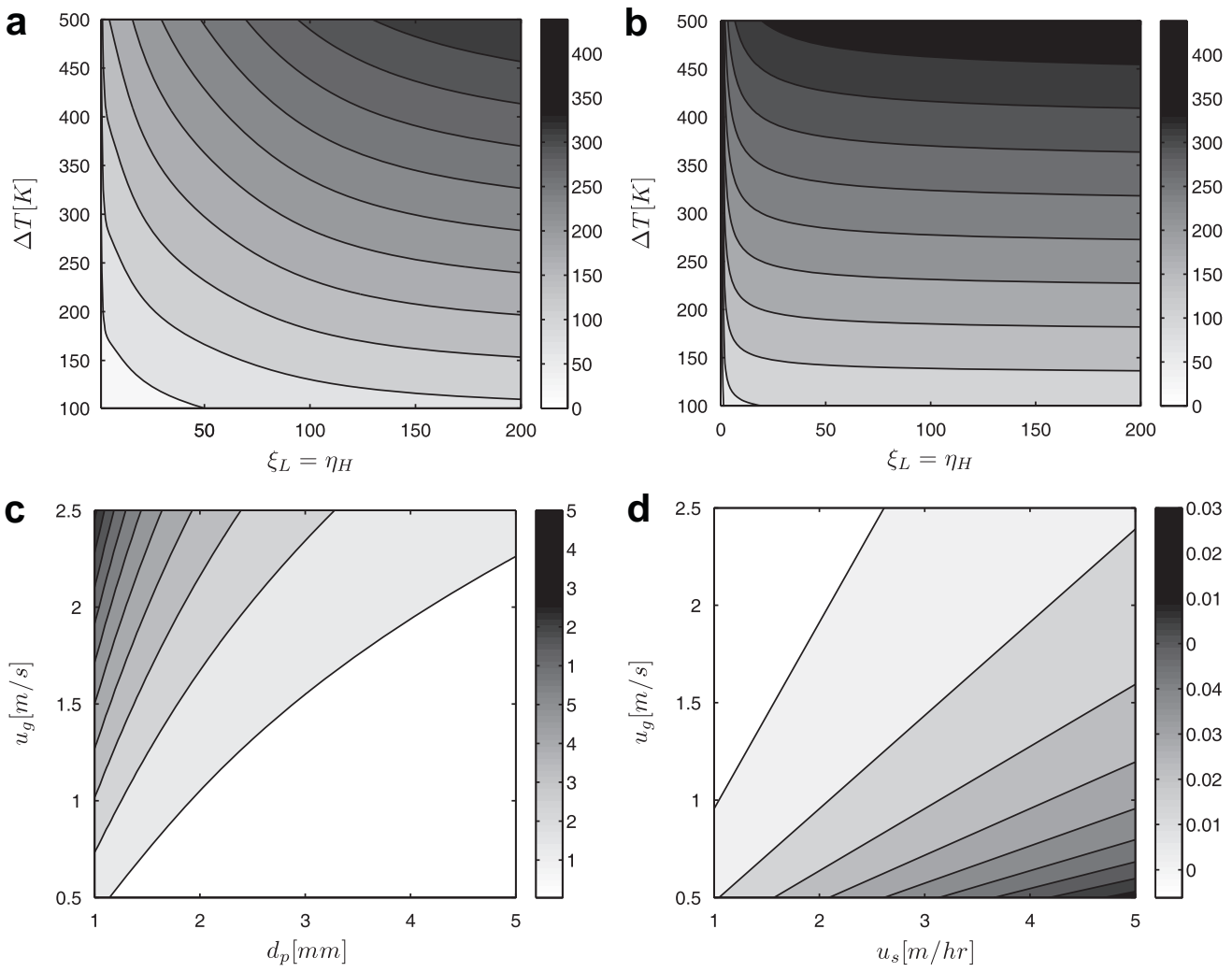

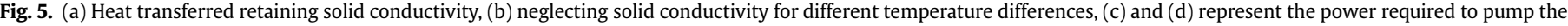
gas and to raise the particles, respectively. The scale is in $\mathrm{kJ} / \mathrm{kg}$.

A quick glimpse at Fig. 5 shows the relative importance of the different energy transfer mechanisms. The power to raise the particles is not relevant, even for high solid velocities. This is in accordance with our previous hypothesis of neglecting solid potential energy variations in Eqs. (1) and (2). The power needed to pump the gas is generally $1-2$ orders of magnitude larger than the necessary power to raise the particles. The heat transfer is, of course, strongly dependant on the available temperature difference between the two flows, but it is always one to two orders of magnitude larger than the power to pump the gas.

Nevertheless, the dependence of the energy variables with the MBHE dimensions was not depicted in Figs. 4 and 5. The required power to pump the gas and to raise the particles both increase linearly with the length of the bed $L$. In contrast, the heat trans ferred has a barely hyperbolic relation with the non dimensional length (in turn directly proportional to the actual length). There fore, for large non dimensional lengths (see Fig. 3(a)), increasing the length of the MBHE will produce a linear increase of the power requirements but with a feeble impact on the heat transferred.

Finally, it should be noted that, being the power values so different between heat transferred and mechanical power requirements, such a feeble impact in heat may prove to add to a heat power increase larger than the power required to further pump the gas. In such cases, the efficiency of blowers and the higher cost of mechanical and/or electrical energy in relation to heat should be taken into account. As a general trend, it seems wise to operate in the zone where the heat transfer reaches a certain stabilization to avoid these larger costs and efficiency based drawbacks. This stabilization point can be established, taking into account the results of Figs. 3 and 5, in the region where the heat exchanger efficiency reaches the $90 \%$. Therefore, an adequate non dimensional length can be defined, as a function of the conduction term incidence, as shown in Fig. 3(b). Thus, a compromise between increasing heat transfer and increasing power requirements and MBHE dimensions should be reached, which is not purely energetic and should consider costs, pump and raiser efficiencies and the relative costs between heat power and electric power.

\section{Conclusions}

The heat transfer analysis of the MBHE shows that large values of solid conductivity in the solid phase reduces the efficiency of the heat exchanger because the width of the region where heat is transferred is augmented, and as a consequence the mean outlet temperature of the cold stream is reduced. Consequently, solids with low conductivity should be selected, although a minimum conductivity is necessary in order to assure that the temperature is uniform in the solid phase, i.e. the Biot number should be lower than 0.1. Thus, to increase the efficiency of a MBHE a compromise between particle size and solid conductivity should be reached fulfilling the limit imposed by the Biot number.

Regarding the other energy interactions (power consumed to pump the gas and to raise the particles), these are orders of magnitude lower than the heat transferred in the range of particle sizes and gas velocities studied and for temperature differences larger than $100 \mathrm{~K}$. Nevertheless, for a proper analysis, the efficiency of the systems employed for pumping the gas and rising the particles and the relative costs between heat and electrical power should be known.

\section{Nomenclature}

$a_{\mathrm{s}} \quad$ superficial area of the particle per unit of volume $\left[\mathrm{m}^{-1}\right]$ $B \quad$ width of the MBHE in the direction perpendicular to both gas and solids flows [m]

Bi Biot number [-] 
specific heat $[\mathrm{J} /(\mathrm{kgK})]$

$d_{\mathrm{p}}$

$\mathrm{H}$ height of the MBHE in the direction of the particle flow $[\mathrm{m}]$

$h_{\mathrm{s}} \quad$ heat transfer coefficient $\left[\mathrm{W} /\left(\mathrm{m}^{2} \mathrm{~K}\right)\right]$

$K \quad$ non dimensional conductivity [-]

$k \quad$ conductivity $[\mathrm{W} /(\mathrm{mK})]$

$L \quad$ length of the MBHE in the direction of the gas flow [m]

$\dot{m} \quad$ mass flow $[\mathrm{kg} / \mathrm{s}]$

MBHE moving bed heat exchanger

$P \quad$ gas pressure [Pa]

$\dot{Q} \quad$ heat transferred in the MBHE [W]

$t \quad$ time [s]

$T \quad$ gas temperature $[\mathrm{K}]$

$\widehat{T} \quad$ non dimensional gas temperature [-]

$\bar{T} \quad$ mean gas temperature $[\mathrm{K}]$

$u \quad$ velocity $[\mathrm{m} / \mathrm{s}]$

$x \quad$ horizontal coordinate (in the direction of the

\section{gas flow) $[\mathrm{m}]$}

$y \quad$ vertical coordinate (in the direction of the solids flow) [m]

$\dot{W}_{g} \quad$ power to pump the gas [W]

$\dot{W}_{\mathrm{s}} \quad$ power to raise the particles [W]

\section{Greek symbols}

$\gamma \quad$ ratio of the gas specific heats [-]

$\Delta P \quad$ gas pressure drop through the MBHE [Pa]

$\varepsilon_{\text {MBHE }} \quad$ efficiency of the MBHE [-]

$\varepsilon \quad$ voidage of the MBHE [-]

$\eta \quad$ non dimensional vertical coordinate $[-]$

$\theta \quad$ solid temperature $[\mathrm{K}]$

$\widehat{\theta} \quad$ non dimensional solid temperature $[-]$

$\bar{\theta} \quad$ mean solid temperature [K]

$\mu \quad$ dynamic viscosity [Pas]

$\xi$ non dimensional horizontal coordinate [-]

$\rho \quad$ density $\left[\mathrm{kg} / \mathrm{m}^{3}\right]$

\section{Subscripts}

g gas phase

in inlet section

max maximum

out outlet section

s solid phase

w at the wall

$\eta \quad$ direction

$\xi \quad$ direction

\section{References}

[1] S.S. Hsiau, J. Smid, F.H. Tsai, J.T. Kuo, C.S. Chou, Placement of flow-corrective elements in a moving granular bed with louvered-walls, Chemical Engineering and Processing 43 (2004) 10371045.

[2] S.S. Hsiau, J. Smid, F.H. Tsai, J.T. Kuo, C.S. Chou, Velocities in moving granular bed filters, Powder Technology 114 (2001) 205212.

[3] S.S. Hsiau, J. Smid, F.H. Tsai, J.T. Kuo, C.S. Chou, Velocity profiles of granules in moving bed filters, Chemical Engineering Science 54 (1999) 293301.

[4] M. Socorro, A. Macias-Machin, J.M. Verona, D. Santana, Hot gas filtration and heat exchange in a packed bed using Lapilli as a granular medium, Industrial and Engineering Chemistry Research 45 (2006) 79577966.
[5] S. Yagi, D. Kunii, Studies on heat transfer near wall surface in packed beds, AIChE Journal 6 (1960) 97104.

[6] S. Yagi, D. Kunii, N. Wakao, Studies on axial effective thermal conductivities in packed beds, AIChE Journal 6 (1960) 543546.

[7] A. Macías-Machín, A. Estevez, J. Cuellar, E. Jaraiz, Simple design of crossflow moving-bed heat exchanger-filter (MHEF), Filtration and Separation 29 (1991) 155161.

[8] E. Achenbach, Heat and flow characteristics of packed beds, Experimental Thermal and Fluid Science 10 (1995) 1727.

[9] A. Henriquez, A. Macias-Machin, Hot gas filtration using a moving bed heat exchanger-filter (MHEF), Chemical Engineering and Processing 36 (1997) 353361.

[10] J.J. Saastamoinen, Heat exchange between two coupled moving beds by fluid flow, International Journal of Heat and Mass Transfer 47 (2004) 15351547.

[11] J.J. Saastamoinen, Comparison of moving bed dryers of solids operating in parallel and counterflow modes, Drying Technology 23 (2005) 10031025.

[12] J. Smid, S.S. Hsiau, C.Y. Peng, H.T. Lee, Moving bed filters for hot gas cleanup, Filtration \& Separation 42 (2005) 3437.

[13] Y.S. Chen, S.S. Hsiau, S.C. Lai, Y.P. Chyoub, H.Y. Li, C.J. Hsua, Filtration of dust particulates with a moving granular bed filter, Journal of hazardous materials 171 (2009) 987994.

[14] J. Smid, S.S. Hsiau, C.Y. Peng, H.T. Lee, Granular moving bed filters and adsorbers (GM-BF/A) - patent review: 1970 2000, Advanced Powder Technology 16 (2005) 301345.

[15] A. Niksiar, A. Rahimi, Design of a moving bed reactor for the production of uranium tetrafluoride based on mathematical modeling, Chemical Engineering Science 65 (2010) 31473157.

[16] Z. Hongjun, S. Mingliang, W. Huixin, L. Zeji, J. Hongbo, Modeling and simulation of moving bed reactor for catalytic naphtha reforming, Petroleum science and technology 28 (2010) 667676.

[17] K. Reijnen, J. van Brakel, Gas cleaning at high temperatures and high pressures: a review, Powder Technology 40 (1984) 81111.

[18] A. Macías-Machín, M. Socorro, J.M. Verona, M. Macías, New granular material for hot gas filtration: use of the Lapilli, Chemical Engineering and Processing 45 (2006) 719727.

[19] A. Soria-Verdugo, J.A. Almendros-Ibánez, U. Ruiz-Rivas, D. Santana, Exergy optimization in a steady moving bed heat exchanger, Annals of the New York Academy of Sciences 1161 (2009) 584600.

[20] G.D.R. Midi, On dense granular flows, European Physical Journal E 14 (2004) 341365.

[21] R.M. Nedderman, C. Laohakul, Thickness of the shear zone of flowing granular-materials, Powder Technology 25 (1980) 91100.

[22] R.P. Zou, A.B. Yu, The packing of spheres in a cylindrical container the thickness effect, Chemical Enginering Science 50 (1995) 15041507.

[23] H. Takahash, H. Yanai, Flow profile and void fraction of granular solids in a moving bed, Powder Technology 7 (1973) 205214.

[24] W. van Antwerpen, C.G. du Toit, P.G. Rousseau, A review of correlations to model the packing structure and effective thermal conductivity in packed beds of mono-sized spherical particles, Nuclear Engineering and Design 240 (2010) 18031818.

[25] M. Giese, K. Rottschafer, D. Vortmeyer, Measured and modeled superficial flow profiles in packed beds with liquid flow, AIChE Journal 44 (1998) 484490.

[26] D. Vortmeyer, R.P. Winter, On the validity limits of packed-bed reactor continuum models with respect to tube to particle diameter ratio, Chemical Engineering Science 39 (1984) 14301432.

[27] Y.S. Teplitskii, V.I. Kovenskii, M.V. Vinogradova, Phenomenological model of heat transfer in an infiltrated granular bed at moderate Reynolds numbers, International Journal of Heat and Mass Transfer 80 (2007) 2122.

[28] Y.H. Chen, X.D. Zhu, Y.Q. Wul, Z. Zhu, Investigation of the effect of a dividing wall in a moving bed, Chemical Engineering and Technology 30 (2007) 10281035.

[29] R. Krupiczka, Analysis of thermal conductivity in granular materials, International Chemical Engineering 7 (1967) 122144.

[30] C.M. Marb, D. Vortmeyer, Multiple steady-states of a cross-flow moving bed reactor: theory and experiment, Chemical Engineering Science 43 (1988) 811819.

[31] D. Vortmeyer, R.J. Schaefer, Equivalence of one-phase and 2-phase models for heat-transfer processes in packed-beds-one-dimensional theory, Chemical Engineering Science 29 (1974) 485491.

[32] J.J. Saastamoinen, Heat exchange between two coupled fixed beds by fluid flow, International Journal of Heat and Mass Transfer 46 (2003) 27272735.

[33] G. Nellis, S. Klein, Heat Transfer. Cambridge University Press, New York, 2009.

[34] S. Ergun, Fluid flow through packed columns, Chemical Engineering Progress 48 (1952) 8994. 